\title{
Incontinentia Pigmenti
}

National Institute of Neurological Disorders and Stroke (NINDS)

\section{Source}

National Institute of Neurological Disorders and Stroke (NINDS). Incontinentia Pigmenti

Information Page.

Incontinentia pigmenti (IP) is an inherited disorder of skin pigmentation that is also associated with abnormalities of the teeth, skeletal system, eyes, and central nervous system. It is one of a group of gene-linked diseases known as neurocutaneous disorders. In most cases, IP is caused by mutations in a gene called NEMO (NF-kappaB essential modulator). Males are more severely affected than females. Discolored skin is caused by excessive deposits of melanin (normal skin pigment). Most newborns with IP will develop discolored skin within the first two weeks. The pigmentation involves the trunk and extremities, is slate-grey, blue or brown, and is distributed in irregular marbled or wavy lines. The discoloration fades with age. Neurological problems include loss of brain tissue (known as cerebral atrophy), the formation of small cavities in the central white matter of the brain, and the loss of neurons in the cerebellar cortex. About $20 \%$ of children with IP will have slow motor development, muscle weakness in one or both sides of the body, impaired cognitive development, and seizures. They are also likely to have visual problems, including crossed eyes, cataracts, and severe visual loss. Dental problems are also common, including missing or peg-shaped teeth. A related disorder, incontinentia pigmenti achromians, features skin patterns of light, unpigmented swirls and streaks that are the reverse of IP. Associated neurological problems are similar. 